\title{
Probing isotopic ratios at redshift $z=0.89$ : molecular line absorption toward PKS 1830-211
}

\author{
S. Muller ${ }^{1,2}$, M. Guélin ${ }^{2}$, M. Dumke ${ }^{3,2}$ and R. Lucas ${ }^{2}$ \\ ${ }^{1}$ Academia Sinica Institute of Astronomy and Astrophysics, P.O. Box 23-141, Taipei, Taiwan \\ email: muller@asiaa.sinica.edu.tw \\ ${ }^{2}$ IRAM, 300 rue de la piscine, 38406 St Martin d'Hères, France \\ ${ }^{3}$ ESO, Alonso de Cordova 3107, Casilla 19001, Santiago 19, Chile
}

\begin{abstract}
Molecular absorption lines in the line of sight to distant quasars are an extremely powerful tool to probe the dense interstellar medium and its chemical composition in intervening galaxies from low to high redshifts. The absorption line measurements of different isotopomers even allow us to study isotopic ratios, which can be interpreted as the signature of past nucleosynthesis activity, and put some constraints on the chemical evolution models.

In this paper, we present the study of molecular absorption lines in front of the quasar PKS 1830-211. The absorption is due to an intervening galaxy at $z=0.89$ which is identified as a nearly face-on spiral galaxy. We have carried out a survey of absorption lines of various $\mathrm{HCO}^{+}, \mathrm{HCN}, \mathrm{HNC}$, and CS isotopomers with the Plateau de Bure Interferometer and derived for the first time the $\mathrm{C}, \mathrm{N}, \mathrm{O}$, and $\mathrm{S}$ isotopic ratios in such a distant object.

This $z=0.89$ absorption system offers an unique opportunity to study the chemical composition in the disk of a spiral galaxy only a few Gyr old. Our results show significantly different isotopic ratios as compared to those measured in the Solar System or in the local ISM, indicating a poorly enhanced abundance of material processed by intermediate and low mass stars.
\end{abstract}

\section{Introduction}

The determination of isotopic ratios in the interstellar medium (ISM) offers a powerful way to investigate past stellar nucleosynthesis and chemical evolution. From mm emission line measurements of different isotopomers, Penzias and co-workers $(1980,1981)$ derived interstellar isotopic ratios and found significant differences between those measured in the Solar System, the local ISM, the Galactic Centre, and in circumstellar envelopes expelled by AGB stars. These differences can be explained to first order by an increasing degree of stellar processing.

This kind of investigation is unfortunately still limited in the case of external galaxies because of the weak emission of rare isotopomers. Only a few results have been reported so far, the most noticeable being the $\mathrm{C}, \mathrm{N}, \mathrm{O}$, and $\mathrm{S}$ ratios in the Large Magellanic Cloud (Chin 1999) and in the nuclei of four nearby starburst galaxies: M82, IC 342, NGC 253, and NGC 4945 (Henkel \& Mauersberger 1993; Henkel et al. 1998; Chin et al. 1999; Wang et al. 2004).

On the other hand, molecular absorption in the line of sight to radio-bright quasars provides a powerful tool to investigate the properties of the dense ISM in distant and young intervening galaxies. The absorption is expressed as $I_{a b s}=I_{0}\left(1-\mathrm{e}^{-\tau}\right)$, where $I_{a b s}$ is the depth of the absorption from the continuum level, $I_{0}$ the flux of the background source, and $\tau$ the optical depth. $\tau$ is proportional to $\mathcal{N} \mu^{2}$, the product of the column 

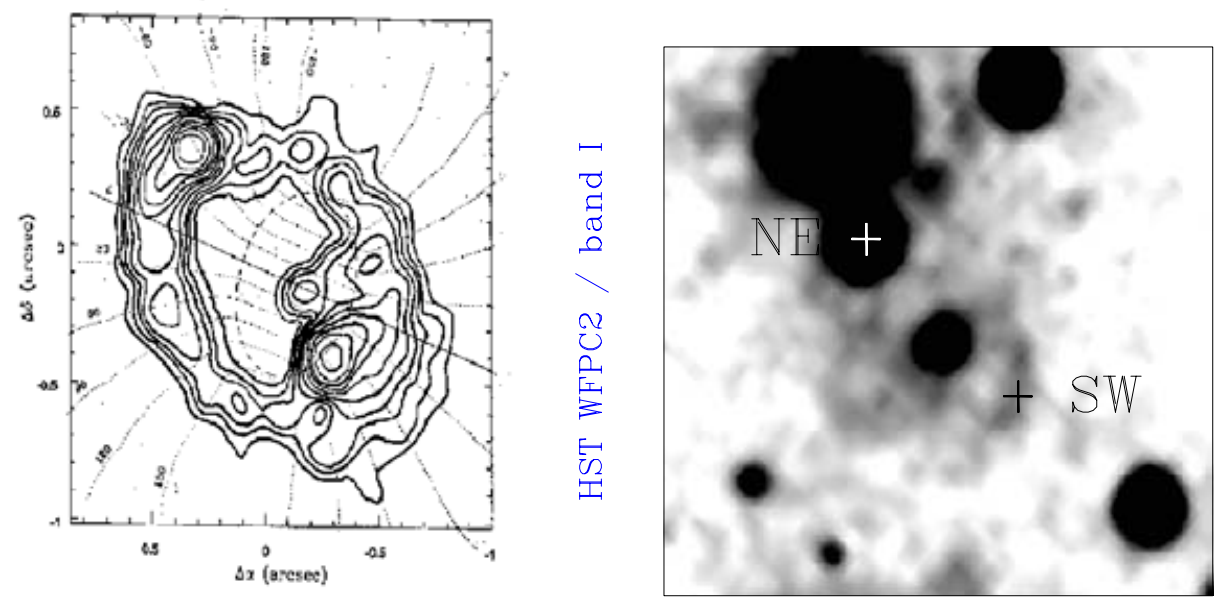

Figure 1. Left: A MERLIN $6 \mathrm{~cm}$ continuum image of PKS 1830, showing the Einstein ring and the 2 brightest lensed images NE and SW of the quasar (from Patnaik et al. 1994). Right: A HST WFPC2 I band image of the $z=0.89$ intervening galaxy (the image has been slightly smoothed to enhance the contrast). The NE and SW positions of the 2 lensed images of the background quasar are indicated. Their separation is $1^{\prime \prime}$.

density $\mathcal{N}$ of the molecule of interest and its permanent electric dipole moment $\mu$. The signal to noise ratio is thus proportional to the continuum flux of the background source, and molecules with large column density and high dipole moment are easier to detect.

Unfortunately, the probability of detecting molecular absorption is lower than in the case of HI $21 \mathrm{~cm}$ or Ly $\alpha$ absorptions because 1) the number of bright quasars is smaller in the millimetre wavelengths, where molecules have their lower J rotational transitions, and 2) the line of sight has to intercept a restricted region, namely the spiral arms in the inner disk of galaxies, where the dense molecular gas is located. As a consequence, the second point goes together with an increased probability of gravitational lensing. Wiklind \& Combes $(1995,1996,1997,1998)$ as well as Menten and co-workers (1998) succeeded in observing a dozen molecular species in the disks of two spiral galaxies with redshifts $z=0.68$ and $z=0.89$, located in front of the gravitationally lensed quasars B0218+357 and PKS 1830-211. Adding to this, molecular lines were also observed in absorption in Cen A (Wiklind \& Combes 1997) and in the galaxies hosting the radio sources PKS 1413+135 ( $z=0.25)$ (Wiklind \& Combes 1994, 1997) and AGN 1504+377 $(z=0.67)$ (Wiklind \& Combes 1996). Nevertheless those pioneering studies did not focus on isotopic ratios.

In this paper, we present the first measurements of the $\mathrm{C}, \mathrm{N}, \mathrm{O}$, and $\mathrm{S}$ isotopic ratios in the spiral arm of a $z=0.89$ galaxy in the line of sight to the quasar PKS 1830-211 (Muller \& Guélin 2003; Muller et al. 2005, in preparation).

\section{The absorption system toward PKS 1830-211}

PKS 1830-211 is a well known gravitational lens, showing a whole Einstein ring connecting two bright images of the quasar at radio wavelengths (Jauncey et al. 1991; Patnaik et al. 1994) as shown in Fig. 1 (left). The two lensed images, hereafter NE and SW, are separated by $1^{\prime \prime}$.

The line of sight to the quasar is intercepted by at least two galaxies: a first one, at a redshift of $z=0.19$, is responsible for narrow HI line absorption, and a second galaxy, 
located at a redshift of $z=0.89$, gives rise to multi-component absorption detected in the lines of numerous molecules (Wiklind \& Combes 1996; Menten et al. 1998).

HST WFPC2 I band deep images have revealed the $z=0.89$ intervening object as a nearly face-on spiral galaxy (Winn et al. 2002) (Fig. 1, right). The two absorption components NE and SW are located on either side of the bulge, each behind a spiral arm. The SW component is closer to the centre of the galaxy at a (model dependent) distance of 2-4 kpc, and presents a larger column density of molecular gas $\left(\mathcal{N}\left(H_{2}\right) \sim 4 \times 10^{22} \mathrm{~cm}^{-2}\right)$.

This geometry provides the opportunity to study the chemical composition of the ISM in the disk of a spiral galaxy only a few Gyr old.

\section{Observations}

We have carried out a survey of absorption lines corresponding to different isotopomers of the $\mathrm{HCO}^{+}, \mathrm{HCN}, \mathrm{HNC}$, and $\mathrm{CS}$ molecules with the Plateau de Bure interferometer (PdBI) toward the $z=0.89$ spiral galaxy in front of the quasar PKS 1830-211.

PKS 1830-211 has a flux between 1.5 and $2 \mathrm{Jy}$ at $3 \mathrm{~mm}$ wavelengths, strong enough to allow the observation of low-opacity lines arising from rare isotopomers and also to selfcalibrate the observations with respect to the continuum emission. Data were obtained between 1999 and 2002. The resulting spectra are presented in Fig. 2.

In the compact configuration of the PdBI, the two components NE and SW are not spatially resolved, so that the spectra of the main isotopomers of $\mathrm{HCO}^{+}, \mathrm{HCN}$, and $\mathrm{HNC}$ present both together the absorption features at $\mathrm{V}_{H E L}=-147 \mathrm{~km} / \mathrm{s}$ and $\mathrm{V}_{H E L}=0 \mathrm{~km} / \mathrm{s}$ respectively. The weaker NE absorption is not detected in the case of the rare isotopomers. The main absorption on the $\mathrm{HCO}^{+}$spectrum appears flat-bottomed between -10 and $10 \mathrm{~km} / \mathrm{s}$, where it reaches an absorption depth of $37 \%$, corresponding to a full absorption of the SW continuum emission. For comparison, the very rare isotopomer $\mathrm{HC}^{17} \mathrm{O}^{+}$only reaches an absorption of $0.55 \%(\tau=0.015)$. The profile of the absorption lines is not symmetric and can be decomposed into several velocity components. Their width of $\sim 10 \mathrm{~km} / \mathrm{s}$ is typical of the widths of molecular clouds in a normal galaxy like our Milky Way.

\section{Isotopic ratios}

From the molecular absorption line measurements, we have derived the isotopic ratios of the $\mathrm{C}, \mathrm{N}, \mathrm{O}$, and $\mathrm{S}$ elements in the $z=0.89$ spiral galaxy. The weak absorption from the rare isotopomers was only detected toward the SW component, and the isotopic ratios therefore only reflect the chemical composition in the spiral arm along this SW beam pencil. Their values are listed in Table 1, where we also give for comparison the corresponding isotopic ratios for a collection of other sources. Fig. 3 shows the same ratios in a more visual form. The isotopic ratios in the $z=0.89$ galaxy differ significantly from those measured in the Solar System and the Milky Way. Except for ${ }^{12} \mathrm{C} /{ }^{13} \mathrm{C}$, whose interpretation is made difficult by optically thick lines of ${ }^{12} \mathrm{C}$, possible fractionation effects and/or selective photo-dissociation, and ${ }^{16} \mathrm{O} /{ }^{17} \mathrm{O}$, they are all on the opposite side of the Solar System values with respect to the ratios observed in IRC +10216 .

The "simple" picture of nucleosynthesis states that ${ }^{12} \mathrm{C}$ and ${ }^{16} \mathrm{O}$ are primary elements produced in massive stars. ${ }^{13} \mathrm{C},{ }^{14} \mathrm{~N}$, and ${ }^{17} \mathrm{O}$ are secondary elements synthesised from ${ }^{12} \mathrm{C}$ and ${ }^{16} \mathrm{O}$ during hydrogen burning in stellar envelopes. ${ }^{15} \mathrm{~N}$ and ${ }^{18} \mathrm{O}$ are produced from explosive $\mathrm{H}$ or He burning. Finally, sulphur is purely an explosive nucleosynthesis product, tracing the high mass stars. The goal here is not to paint a whole scenario of the evolution of the nuclear processing, but we can observe some tendencies. The ${ }^{18} \mathrm{O} /{ }^{17} \mathrm{O}$ and ${ }^{14} \mathrm{~N} /{ }^{15} \mathrm{~N}$ ratios, for example, are found to be high and low respectively, which is 

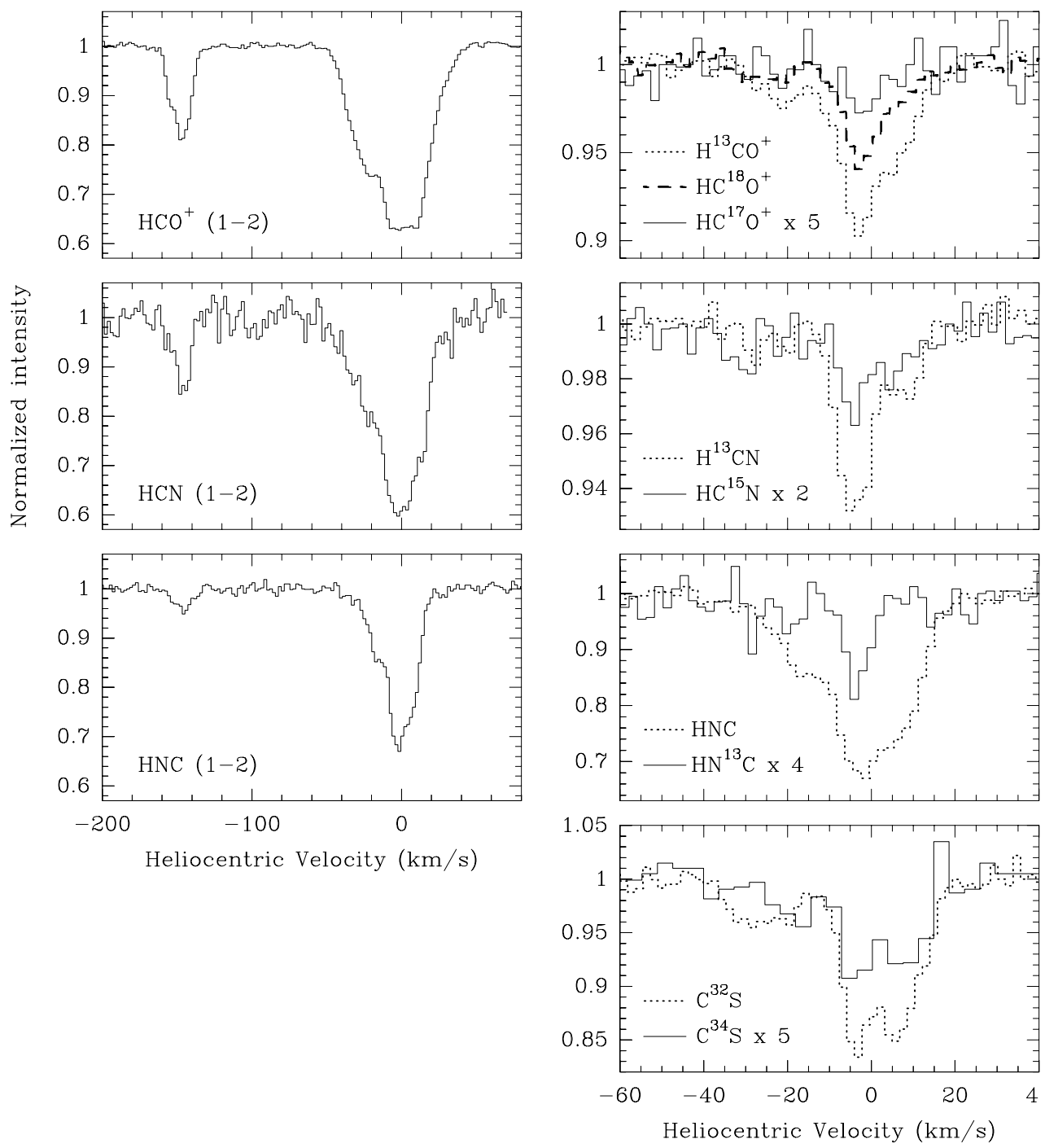

Figure 2. Absorption spectra of the different isotopomers of $\mathrm{HCO}^{+}(1-2), \mathrm{HCN}(1-2), \mathrm{HNC}(1-2)$, and $\mathrm{CS}(3-4)$ as observed with the Plateau de Bure interferometer. $\mathrm{V}_{H E L}=0 \mathrm{~km} / \mathrm{s}$ corresponds to $z=0.88582$. The main isotopomers (left column) show the absorption of the 2 components: $\mathrm{SW}$, at $\mathrm{V}_{H E L}=0 \mathrm{~km} / \mathrm{s}$ and NE, at $\mathrm{V}_{H E L}=-147 \mathrm{~km} / \mathrm{s}$. For the rare isotopomers (right column), the absorption is only detected toward the SW component. The velocity resolution is $2 \mathrm{~km} / \mathrm{s}$ except for the spectrum of $\mathrm{C}^{34} \mathrm{~S}$, for which it is $4 \mathrm{~km} / \mathrm{s}$.

consistent with the idea that in such a young object only a few Gyr old (look-back time of $\sim 6 \mathrm{Gyr}$ ), low mass stars had not had sufficient time to play a role in the regeneration and processing of the interstellar gas. Similarly, the measured ${ }^{32} \mathrm{~S} /{ }^{34} \mathrm{~S}$ ratio $(10 \pm 1)$ is twice as small as the value observed in the Solar System, in IRC +10216 , and in the local ISM, again suggesting a nucleosynthesis history different from that of the Milky Way.

It is interesting to notice that the closer isotopic ratios are found in the nuclei of nearby starburst galaxies, where the outputs from short living massive stars should also 


\begin{tabular}{|c|c|c|c|c|c|c|c|}
\hline Isotopic ratio & ${ }^{\mathbf{1 2}} \mathrm{C} /{ }^{\mathbf{1 3}} \mathrm{C}$ & ${ }^{14} \mathrm{~N} /{ }^{15} \mathrm{~N}$ & ${ }^{16} \mathrm{O} /{ }^{18} \mathrm{O}$ & ${ }^{16} \mathrm{O} /{ }^{17} \mathrm{O}$ & ${ }^{18} \mathrm{O} /{ }^{17} \mathrm{O}$ & ${ }^{32} \mathrm{~S} /{ }^{34} \mathrm{~S}$ \\
\hline $\boldsymbol{z}=\mathbf{0 . 8 9}$ galaxy & $\mathbf{3 2} \pm \mathbf{1 0}$ & $\mathbf{1 4 0} \mathbf{- 5 0}_{-50}^{+130}$ & $\mathbf{6 7} \pm \mathbf{2 4}$ & $\mathbf{8 7 1} \pm \mathbf{5 1 3}$ & $\mathbf{1 3} \pm \mathbf{3}$ & $\mathbf{1 0} \pm \mathbf{1}$ \\
LMC (a) & $62 \pm 5$ & $114 \pm 14$ & $>2000$ & $>3600$ & $1.8 \pm 0.4$ & $18 \pm 6$ \\
Solar System (b) & 89 & 270 & 490 & 2695 & 5.5 & 22 \\
Local ISM (c) & $59 \pm 2$ & $237_{-21}^{+27}$ & $672 \pm 110$ & $2352 \pm 721$ & $3.5 \pm 0.5$ & $19 \pm 8$ \\
Galactic Centre (b) & $25 \pm 5$ & $900 \pm 200$ & $250 \pm 30$ & $875 \pm 155$ & $3.5 \pm 0.2$ & $18 \pm 5$ \\
NGC 4945 (d) & $50 \pm 10$ & $105 \pm 25$ & $195 \pm 45$ & $1248 \pm 347$ & $6.4 \pm 0.3$ & $13.5 \pm 2.5$ \\
IRC+10216 (e) & $45 \pm 3$ & $>4400$ & $1260_{-240}^{+315}$ & $882 \pm-473$ & $0.7 \pm 0.2$ & $21.8 \pm 2.6$ \\
\hline
\end{tabular}

Table 1. C, N, O, and S isotopic ratios in the $z=0.89$ galaxy, the Large Magellanic Cloud (LMC), the Solar System, the local ISM, the Galactic centre, the nuclei of the nearby starburst galaxy NGC 4945, and the envelope of the AGB star IRC+10216.

References: a) Chin (1999); b) Wilson \& Rood (1994), and references therein; c) Lucas \& Liszt (1998), and Penzias (1981); d) Wang et al. (2004); e) Kahane et al. (1988, 1992), and Cernicharo et al. (2000).

dominate. On the other hand, there are some differences with the isotopic ratios measured in the LMC, which may not be expected if the two objects share a common low metallicity.

\section{Conclusions}

Molecular absorption lines in front of radio-bright quasars remain a unique tool to investigate the properties of the dense interstellar medium in distant galaxies. One particular application is the determination of the isotopic ratios in these young objects, providing information about nuclear processing and chemical evolution.

We have presented here millimetre absorption lines in a $z=0.89$ spiral galaxy located in the line of sight to the quasar PKS 1830-211. C, N, O, and S isotopic ratios were derived for the first time in such a young and distant galaxy from observations of different isotopomers of the $\mathrm{HCO}^{+}, \mathrm{HCN}, \mathrm{HNC}$, and $\mathrm{CS}$ molecules. The results are consistent with the image of a young interstellar medium, poorly processed and with a low enrichment from low and intermediate mass stars.

While this work provides some observational constraints for chemical evolution models, there is no doubt that ALMA will play a major role in this field by allowing us to extend this kind of study to a large number of objects over a wide range of redshifts and chemical evolution states.

\section{Acknowledgements}

S. M. is very grateful to the LOC for giving the opportunity to present this work, and for an IAU grant of financial support.

\section{References}

Carilli, C. L., et al., 1998, astro-ph/9801157

Cernicharo, J., Guélin, M., Kahane, C., 2000, A\&AS, 142, 181

Chin, Y.-N., et al., 1999, ApJ, 512, L143

Chin, Y.-N., 1999, IAU Symp. 190, 279

Combes, F., Wiklind, T., 1998, ESO Messenger, 91, 29

Harrison, A., Henkel, C., Russell, A., 1999 MNRAS, 303, 157

Henkel, C., et al., 1998, A\&A, 329, 443

Henkel, C., Mauersberger, R., 1993, A\&A, 274, 730

Jauncey, D. L., et al., 1991, Nature, 352, 132 

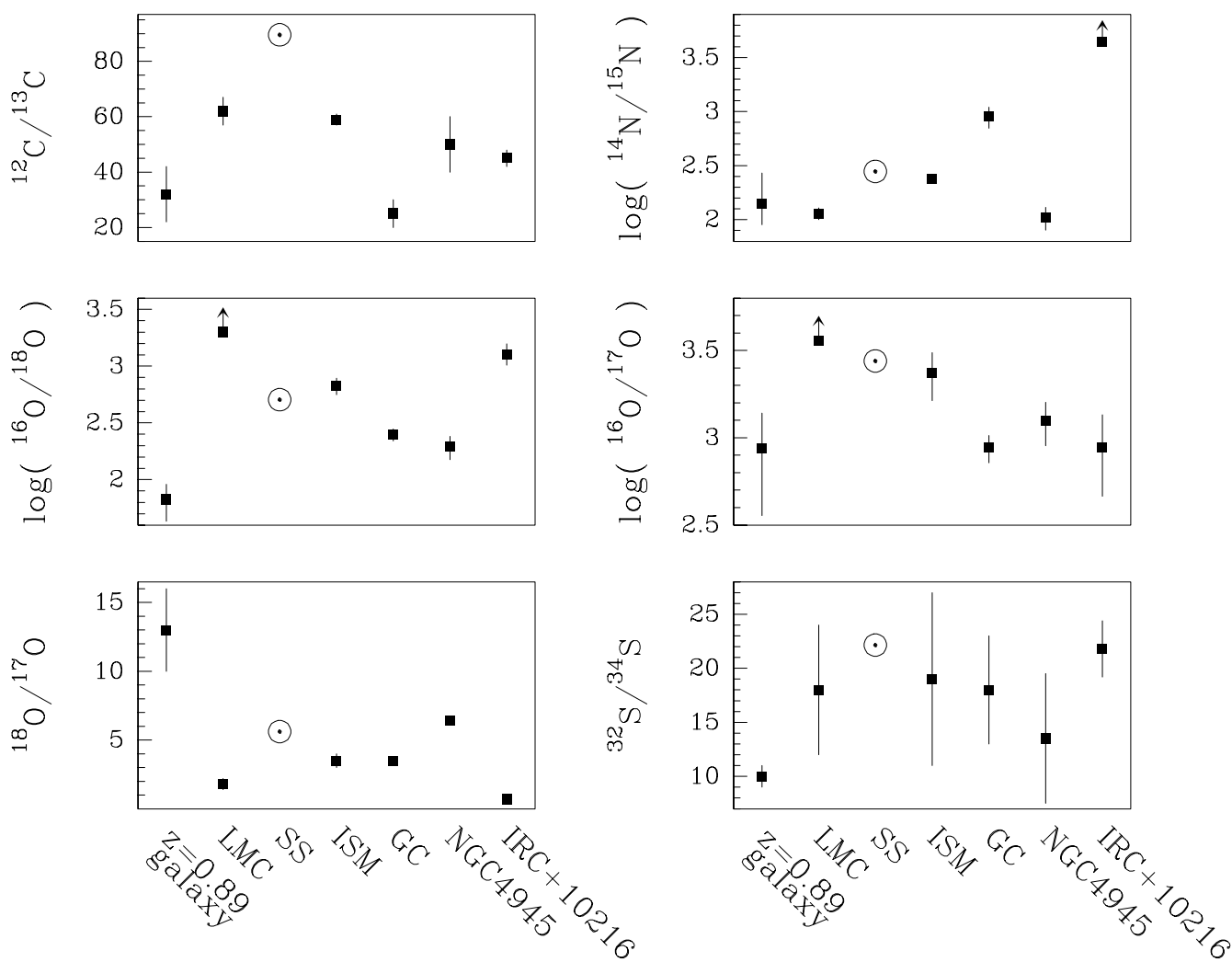

Figure 3. Comparison of the isotopic ratios in the $z=0.89$ galaxy, the Large Magellanic Cloud (LMC), the Solar System (SS), the local interstellar medium (ISM), the Galactic Centre (GC), the nucleus of the nearby starburst galaxy NGC 4945, and the circumstellar envelope of the AGB star IRC+10216. The corresponding references are indicated in the legend of Table 1.

Kahane, C., et al., 1988, A\&A, 190, 167

Lucas, R., Liszt, H., 1998, A\&A, 337, 246

Martin, S., et al., 2005, ApJ, 620, 210

Menten, K. M., Carilli, C. L., Reid, M. J., 1998, astro-ph/9812178

Muller, S., Guélin, M., 2003, ASP Conf. Series, 304, 252

Muller, S., Guélin, M., Dumke, M., Lucas, R., 2005, to be submitted to A\&A

Patnaik, A., et al., 1994, in Proceedings of the 1993 Liege Symposium on Gravitational Lensing

Penzias, A. A., 1980, Science, 208, 663

Penzias, A. A., 1981, ApJ, 249, 518

Wang, M., et al., 2004, A\&A, 422, 883

Wiklind, T., Combes, F., 1994, A\&A, 286, L9

Wiklind, T., Combes, F., 1995, A\&A, 299, 382

Wiklind, T., Combes, F., 1996, Nature, 379, 139

Wiklind, T., Combes, F., 1996, A\&A, 315, 84

Wiklind, T., Combes, F., 1997, A\&A, 324, 51

Wiklind, T., Combes, F., 1997, A\&A, 328, 48

Wiklind, T., Combes, F., 1998, ApJ, 500, 129

Wilson, T. L., Rood, R. T., 1994, ARA\&A, 32, 191

Winn, J. N., et al., 2002, ApJ, 575, 103 